\title{
Comparative Analysis of Newmark and Rungekutta Method for Solving A TBM Cutterhead Multi-DOF Dynamic Model
}

\author{
Junzhou Huo ${ }^{1}$, Hanyang $\mathrm{Wu}^{1}$, Jing Chen ${ }^{2 *}$, Jingxiu Ling ${ }^{1}$ and Guangqing $\mathrm{Li}^{1}$ \\ ${ }^{1}$ School of Mechanical Engineering, Dalian University of Technology, \\ Dalian116024, China \\ ${ }^{2}$ School of Naval Architecture and Ocean Engineering, Dalian Ocean University, \\ Dalian 116023, P.R. China \\ 2684822837@qq.com
}

\begin{abstract}
In engineering, solving divergence and long solving time often occurs when solving large complex dynamic equations. Comparative analysis of Newmark and Rungekutta method fora complex dynamic model can provide a theory basis for the selection of numerical integration method when solving complex dynamic equation. The comparative analysis on the solving precision and solving time by Newmark method and Rungekutta method for calculating a same complex dynamic equation is carried out. The calculation results show that the response results by these two methods are in strong consistency. But the Newmark method shortens the solving time by at least 3 orders comparing that of Rungekutta method. Furthermore, the response displacement by Newmark method in different integration steps is obtained. The results indicate that the response results become stable with integration step continues reducing under 0.1 .
\end{abstract}

Keywords: Newmark method; dynamic equations; solving time

\section{Introduction}

In engineering, the dynamic characteristics of each component are analyzed by solving the corresponding dynamic equations. The dynamic equations will become time-varying and nonlinear when considering the time-varying stiffness and multi-DOF coupling structure. Therefore, solving divergence and long solving time are the common problems in the calculation process.

The foreign and domestic scholars have done lots of researches on Newmark numerical integration method for solving dynamic equations. Some scholars carried out theoretical derivation for deeper analyzing the precision volatility problem ${ }^{[2]}$. Some put forward a method that can automatically adjust the integration step based on the original Newmark method ${ }^{[3,4]}$. Newmark and Rungekutta method are the most used numerical integral method for calculating large complex dynamic equations. Many scholars have done deep researches on the solving precision and stability of Newmark method, Rungekutta method, etc ${ }^{[5]-[8]}$. But the comparative analysis on the solving precision and solving time by Newmark method, Rungekutta method for a same complex dynamic equations is still not that deep.

TBM cutterhead multi-DOF dynamic model

This paper takes a TBM cutterhead complex dynamic model as an example [9], and the dynamic responses of cutterhead system are obtained by using Newmark method. The 
Coupled nonlinear dynamic model of TBM cutterhead system is shown in figure 1 . The dynamic model contains 59 DOF totally. There are 20 DOF in torsional direction while radial direction contains $26 \mathrm{DOF}$, and axis and pendulum coupled direction contains 14 DOF.

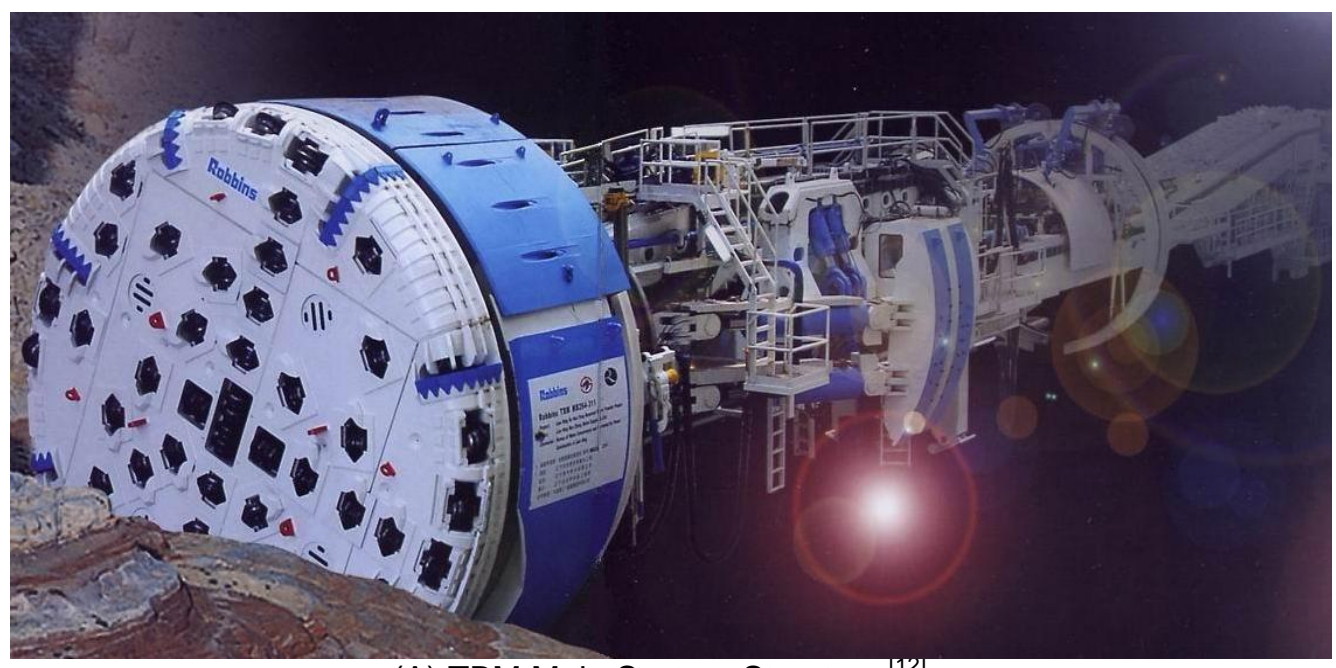

(A) TBM Main System Structure ${ }^{[12}$
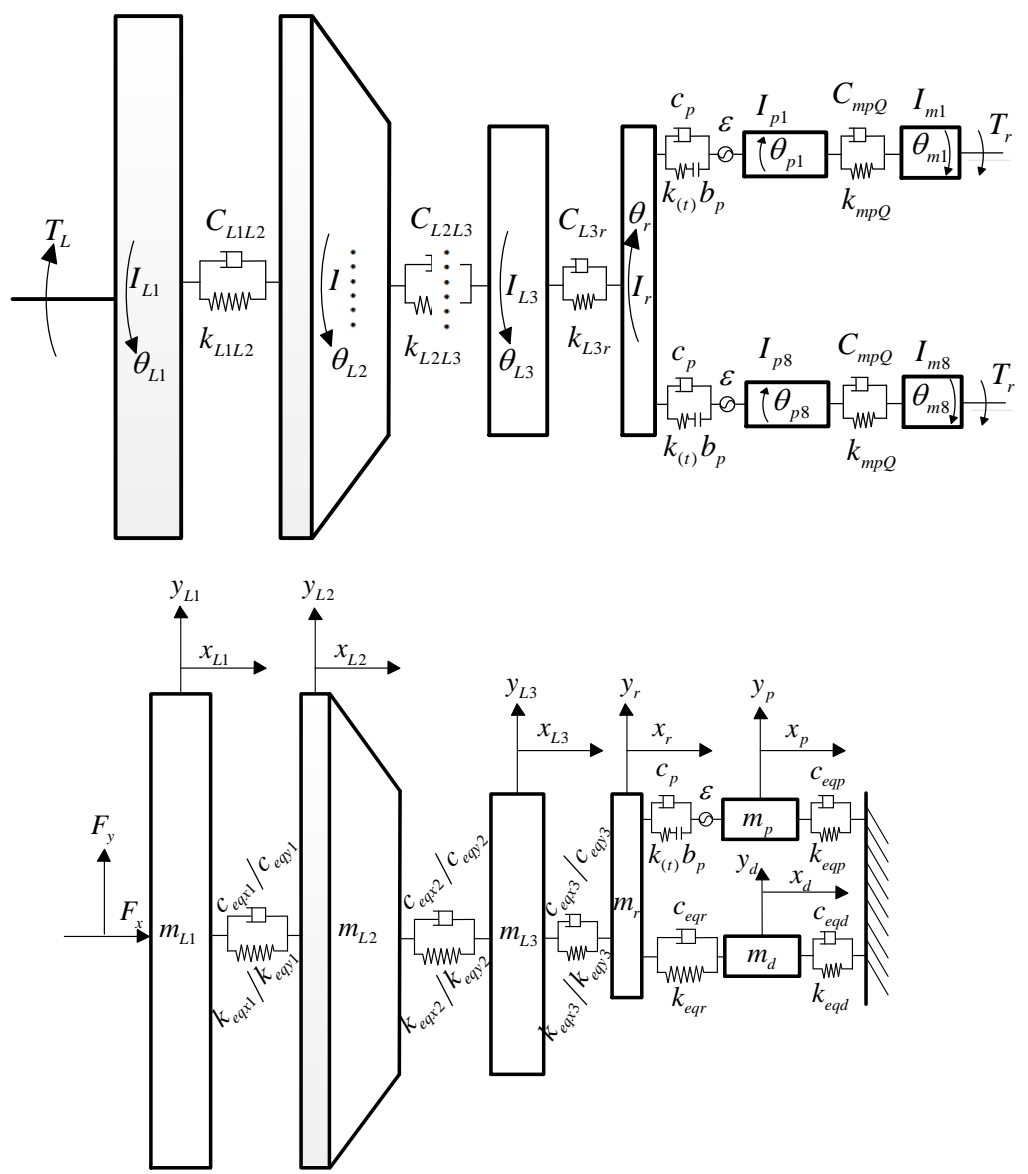

(B) Torsional Coupledcutterhead System Dynamics Model 

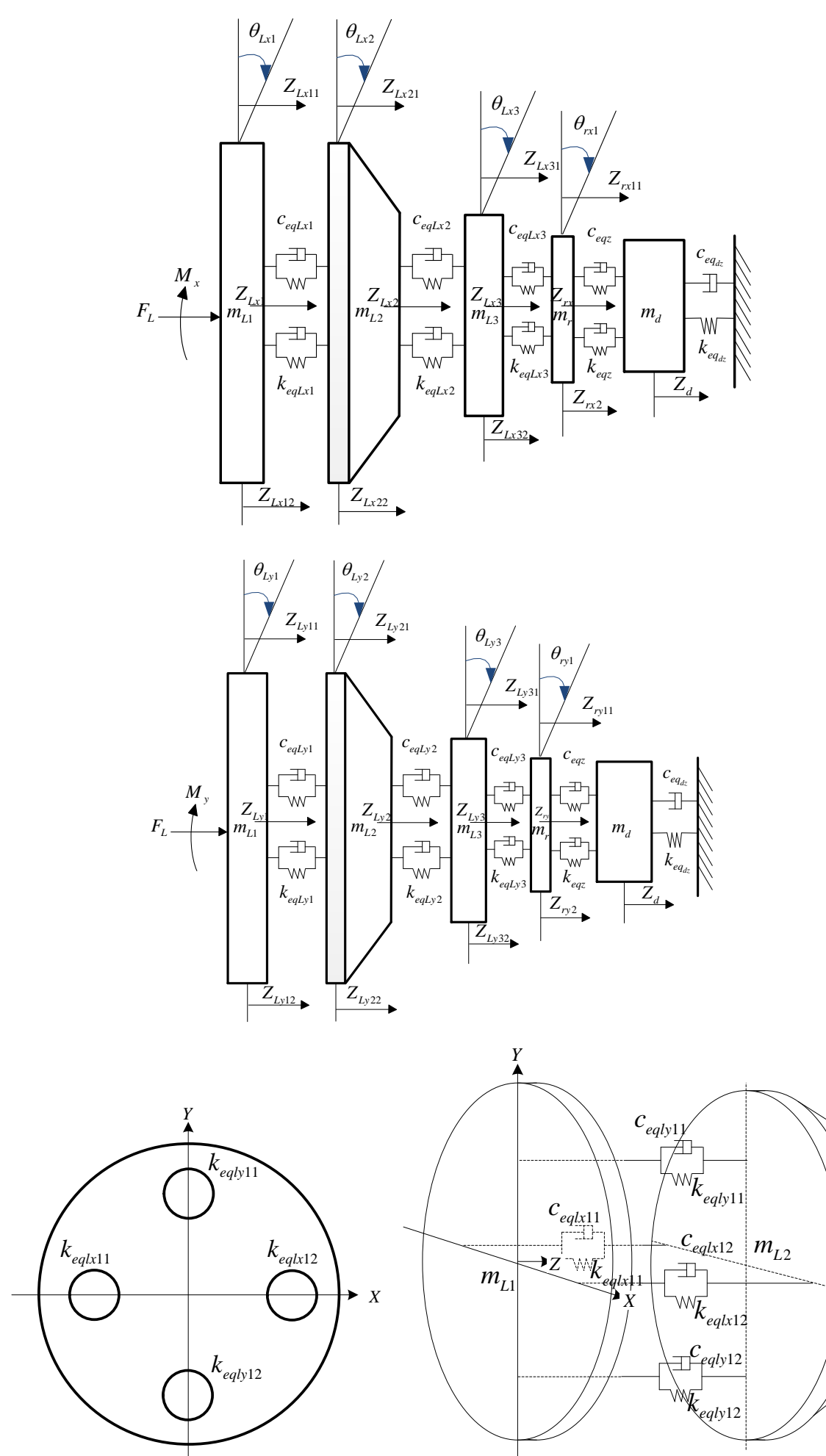

(C) Axis and Pendulum Coupled Cutterhead System Dynamics Model

Figure 1. Coupled Nonlinear Dynamic Model of Tbm Cutterhead System 
The dynamic equations are listed below:

$$
\begin{aligned}
& \left\{\begin{array}{l}
I_{m i} \ddot{\theta}_{m i}+C_{m p Q}\left(\dot{\theta}_{m i}-\dot{\theta}_{p i}\right)+k_{m p Q}\left(\theta_{m i}-\theta_{p i}\right)=T_{p i} \\
I_{p i} \ddot{\theta}_{p i}+\left(F_{p r i}+D_{p r i}\right) r_{b p}+C_{m p Q}\left(\dot{\theta}_{p i}-\dot{\theta}_{m i}\right)+k_{m p Q}\left(\theta_{p i}-\theta_{m i}\right)=0 \\
I_{r} \ddot{\theta}_{r}-\sum_{i=1}^{N}\left(F_{p r i}+D_{p r i}\right) r_{b r}+C_{r L 3}\left(\dot{\theta}_{r}-\dot{\theta}_{L 3}\right)+k_{r L 3}\left(\theta_{r}-\theta_{L 3}\right)=0 \\
I_{L 3} \ddot{\theta}_{L 3}+C_{L 3 r}\left(\theta_{L 3}-\theta_{r}\right)+k_{L 3 r}\left(\theta_{L 3}-\theta_{r}\right)+C_{L 2 L 3}\left(\theta_{L 3}-\theta_{L 2}\right)+k_{L 2 L 3}\left(\theta_{L 3}-\theta_{L 2}\right)=0 \\
I_{L 2} \ddot{\theta}_{L 2}+C_{L 2 L 3}\left(\theta_{L 2}-\theta_{L 3}\right)+k_{L 2 L 3}\left(\theta_{L 2}-\theta_{L 3}\right)+C_{L 1 L 2}\left(\theta_{L 2}-\theta_{L 1}\right)+k_{L 1 L 2}\left(\theta_{L 2}-\theta_{L 1}\right)=0 \\
I_{L 1} \ddot{\theta}_{L 1}+C_{L 1 L 2}\left(\theta_{L 1}-\theta_{L 2}\right)+k_{L 1 L 2}\left(\theta_{L 1}-\theta_{L 2}\right)=-T_{L}
\end{array}\right. \\
& \left(\begin{array}{l}
m_{L 1} \ddot{X}_{L 1}+C_{e q 11}\left(\dot{X}_{L 1}-\dot{X}_{L 2}\right)+k_{e q x 1}\left(X_{L 1}-X_{L 2}\right)=F_{X} \\
m_{L 1} \ddot{Y}_{L 1}+C_{e q 11}\left(\dot{Y}_{L 1}-\dot{Y}_{L 2}\right)+k_{e q 11}\left(Y_{L 1}-Y_{L 2}\right)=F_{Y}
\end{array}\right. \\
& m_{L 2} \ddot{X}_{L 2}+C_{\text {equ1 } 1}\left(\dot{X}_{L 2}-\dot{X}_{L 1}\right)+C_{\text {eq } 2}\left(\dot{X}_{L 2}-\dot{X}_{L 3}\right)+k_{\text {equ1 } 1}\left(X_{L 2}-X_{L 1}\right)+k_{\text {eqq2 } 2}\left(X_{L 2}-X_{L 3}\right)=0 \\
& m_{L 2} \ddot{Y}_{L 2}+C_{\text {eqy } 1}\left(\dot{Y}_{L 2}-\dot{Y}_{L 1}\right)+C_{e q q 2}\left(\dot{Y}_{L 2}-\dot{Y}_{L 3}\right)+k_{\text {eqy } 1}\left(Y_{L 2}-Y_{L 1}\right)+k_{\text {eq } 2}\left(Y_{L 2}-Y_{L 3}\right)=0 \\
& m_{L 3} \ddot{X}_{L 3}+C_{\text {eqx } 2}\left(\dot{X}_{L 3}-\dot{X}_{L 2}\right)+C_{\text {equ3 } 3}\left(\dot{X}_{L 3}-\dot{X}_{r}\right)+k_{\text {eqq2 } 2}\left(X_{L 3}-X_{L 2}\right)+k_{\text {eqx } 3}\left(X_{L 3}-X_{r}\right)=0 \\
& m_{L 3} \ddot{Y}_{L 3}+C_{\text {eq } 2}\left(\dot{Y}_{L 3}-\dot{Y}_{L 2}\right)+C_{\text {eqy } 3}\left(\dot{Y}_{L 3}-\dot{Y}_{r}\right)+k_{\text {eqy } 2}\left(Y_{L 3}-Y_{L 2}\right)+k_{\text {equ } 3}\left(Y_{L 3}-Y_{r}\right)=01 \\
& m_{r} \ddot{X}_{r}+\sum_{i=1}^{N}\left(F_{p r i}+D_{p r i}\right) \sin \left(\varphi_{i}+\alpha\right)+C_{e q r}\left(\dot{X}_{r}-\dot{X}_{d}\right)+C_{e q 93}\left(\dot{X}_{r}-\dot{X}_{L 3}\right)+k_{\text {eqr }}\left(X_{r}-X_{d}\right)+k_{e q u 3}\left(X_{r}-X_{L 3}\right)=0 \\
& m_{r} \ddot{Y}_{r}-\sum_{i=1}^{N}\left(F_{p r i}+D_{p r i}\right) \cos \left(\varphi_{i}+\alpha\right)+C_{e q r}\left(\dot{Y}_{r}-\dot{Y}_{d}\right)+C_{e q 93}\left(\dot{Y}_{r}-\dot{Y}_{L 3}\right)+k_{e q r}\left(Y_{r}-Y_{d}\right)+k_{e q g 3}\left(Y_{r}-Y_{L 3}\right)=0 \\
& m_{p i} \ddot{X}_{p i}+\left(F_{p r i}+D_{p r i}\right) \cos \alpha+C_{e p p i} \dot{X}_{p i}+k_{e q p i} X_{p i}=0 \\
& m_{p i} \ddot{Y}_{p i}+\left(F_{p r i}+D_{p r i}\right) \sin \alpha+C_{\text {eppi }} \dot{Y}_{p i}+k_{\text {eqpi }} Y_{p i}=0 \\
& m_{d} \ddot{X}_{d}+C_{e q r}\left(\dot{X}_{d}-\dot{X}_{r}\right)+C_{e q d} \dot{X}_{d}+k_{e q r}\left(X_{d}-X_{r}\right)+k_{\text {eqd }} X_{d}=0 \\
& m_{d} \ddot{Y}_{d}+C_{\text {eqr }}\left(\dot{Y}_{d}-\dot{Y}_{r}\right)+C_{\text {eqd }} \dot{Y}_{d}+k_{\text {eqr }}\left(Y_{d}-Y_{r}\right)+k_{\text {eqd }} Y_{d}=0
\end{aligned}
$$




$$
\begin{aligned}
& \left(m_{L 1} \ddot{Z}_{L 1}+C_{e q L x 11}\left(\dot{Z}_{L x 11}-\dot{Z}_{L x 21}\right)+C_{e q L x 12}\left(\dot{Z}_{L x 12}-\dot{Z}_{L x 22}\right)+C_{e q L y 11}\left(\dot{Z}_{L y 11}-\dot{Z}_{L y 21}\right)+C_{e q L y 12}\left(\dot{Z}_{L y 12}-\dot{Z}_{L y 22}\right)\right. \\
& +k_{e q L x 11}\left(Z_{L x \times 11}-Z_{L x \times 1}\right)+k_{e q L x 12}\left(Z_{L x 12}-Z_{L x 22}\right)+k_{e q L y 11}\left(Z_{L y 11}-Z_{L y 21}\right)+k_{e q L y 12}\left(Z_{L y y 12}-Z_{L y 22}\right)=F_{L} \\
& m_{L 2} \ddot{Z}_{L 2}+C_{e q L x 11}\left(\dot{Z}_{L x 21}-\dot{Z}_{L x 11}\right)+C_{e q L x 12}\left(\dot{Z}_{L x 22}-\dot{Z}_{L x 12}\right)+C_{e q L y 11}\left(\dot{Z}_{L y 21}-\dot{Z}_{L y 11}\right)+C_{e q L y 12}\left(\dot{Z}_{L y 22}-\dot{Z}_{L y 12}\right) \\
& +C_{e q L x 21}\left(\dot{Z}_{L x 21}-\dot{Z}_{L x 31}\right)+C_{e q L x 22}\left(\dot{Z}_{L x 22}-\dot{Z}_{L x 32}\right)+C_{e q L y 21}\left(\dot{Z}_{L y 21}-\dot{Z}_{L y 31}\right)+C_{e q L y 22}\left(\dot{Z}_{L y 22}-\dot{Z}_{L y 32}\right) \\
& +k_{e q L x 11}\left(Z_{L x 21}-Z_{L x 11}\right)+k_{e q L x 12}\left(Z_{L x 22}-Z_{L x 12}\right)+k_{e q L y \mid 11}\left(Z_{L y 21}-Z_{L y y 11}\right)+k_{e q L y 12}\left(Z_{L y 22}-Z_{L y 12}\right) \\
& +k_{e q L x 21}\left(Z_{L x 21}-\dot{Z}_{L x 31}\right)+k_{e q L x 22}\left(Z_{L x 22}-Z_{L x 32}\right)+k_{e q L y y 21}\left(Z_{L y 21}-Z_{L y 31}\right)+k_{e q L y 22}\left(Z_{L y 22}-Z_{L y 32}\right)=0 \\
& m_{L 3} \ddot{Z}_{L 3}+C_{e q L L x 21}\left(\dot{Z}_{L x 31}-\dot{Z}_{L x 21}\right)+C_{e q L x 222}\left(\dot{Z}_{L x 32}-\dot{Z}_{L x 222}\right)+C_{e q L y 21}\left(\dot{Z}_{L y 31}-\dot{Z}_{L y y 21}\right)+C_{e q L L y 22}\left(\dot{Z}_{L y 332}-\dot{Z}_{L y 22}\right) \\
& +C_{e q L x 31}\left(\dot{Z}_{L x 31}-\dot{Z}_{r x 1}\right)+C_{e q L x 32}\left(\dot{Z}_{L x 32}-\dot{Z}_{r x 2}\right)+C_{e q L y 31}\left(\dot{Z}_{L y 31}-\dot{Z}_{r y 1}\right)+C_{e q L \zeta 32}\left(\dot{Z}_{L y 332}-\dot{Z}_{r 22}\right) \\
& +k_{e q L x 21}\left(Z_{L x 31}-Z_{L x 21}\right)+k_{e q L x 22}\left(Z_{L x 32}-Z_{L x 22}\right)+k_{e q L y 21}\left(Z_{L y 31}-Z_{L y 21}\right)+k_{e q L y 22}\left(Z_{L y 32}-Z_{L y 22}\right) \\
& +k_{e q L x>1}\left(Z_{L x 31}-Z_{r x 1}\right)+k_{e q L x 32}\left(Z_{L x 32}-Z_{r x 2}\right)+k_{e q L y 31}\left(Z_{L y 31}-Z_{r y 1}\right)+k_{e q L Y \zeta 32}\left(Z_{L y 32}-Z_{r y 2}\right)=0 \\
& m_{r} \ddot{Z}_{r}+C_{e q L x 31}\left(\dot{Z}_{r x 1}-\dot{Z}_{L x 31}\right)+C_{e q L x 32}\left(\dot{Z}_{r x 2}-\dot{Z}_{L x 32}\right)+C_{e q L y 31}\left(\dot{Z}_{r y 1}-\dot{Z}_{L y 31}\right)+C_{e q L y 32}\left(\dot{Z}_{r y 2}-\dot{Z}_{L y 32}\right) \\
& +C_{e q x x 1}\left(\dot{Z}_{r x 1}-\dot{Z}_{d}\right)+C_{e q x x 2}\left(\dot{Z}_{r x 2}-\dot{Z}_{d}\right)+C_{e q q y 1}\left(\dot{Z}_{r y 1}-\dot{Z}_{d}\right)+C_{e q y 2}\left(\dot{Z}_{r y 2}-\dot{Z}_{d}\right) \\
& +k_{e q L x 31}\left(Z_{r x 1}-Z_{L x 31}\right)+k_{e q L x 32}\left(Z_{r x 2}-Z_{L x 32}\right)+k_{e q L y 31}\left(Z_{r y 1}-Z_{L y 31}\right)+k_{e q L y 32}\left(Z_{r y 2}-Z_{L y 32}\right) \\
& +k_{\text {eqxx1 }}\left(Z_{r x 1}-Z_{d}\right)+k_{e q x \times 2}\left(Z_{r x 2}-Z_{d}\right)+k_{e q y y 1}\left(Z_{r y 1}-Z_{d}\right)+k_{e q y 2}\left(Z_{r y 2}-Z_{d}\right)=0 \\
& m_{d} \ddot{Z}_{d}+C_{e q z x 1}\left(\dot{Z}_{d}-\dot{Z}_{r x 1}\right)+C_{e q 7 x 2}\left(\dot{Z}_{d}-\dot{Z}_{r x 2}\right)+C_{e q z y 11}\left(\dot{Z}_{d}-\dot{Z}_{r y 1}\right)+C_{e q 7 y 2}\left(\dot{Z}_{d}-\dot{Z}_{r y 2}\right)+C_{e q d z} \dot{Z}_{d}
\end{aligned}
$$

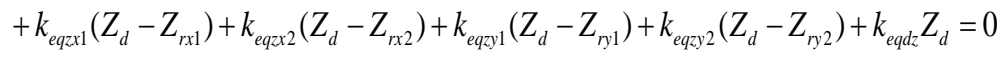

$$
\begin{aligned}
& I_{L 1 x} \ddot{\theta}_{L 1 x}+C_{e q L x 11}\left(\dot{Z}_{L x 11}-\dot{Z}_{L x 21}\right) a_{L 1}-C_{e q L x 12}\left(\dot{Z}_{L \times 12}-\dot{Z}_{L x 22}\right) a_{L 1}+k_{e q L x 11}\left(Z_{L x 11}-Z_{L x 21}\right) a_{L 1}-k_{e q L x 12}\left(Z_{L x 12}-Z_{L x 22}\right) a_{L 1}=M_{X} \\
& I_{L 1 y} \ddot{\theta}_{L 1 y}+C_{e q L y 11}\left(\dot{Z}_{L y 11}-\dot{Z}_{L y 21}\right) a_{L 1}-C_{e q L L 12}\left(\dot{Z}_{L y 12}-\dot{Z}_{L y 22}\right) a_{L 1}+k_{e q L y 11}\left(Z_{L y y 11}-Z_{L y 21}\right) a_{L 1}-k_{e q L y 12}\left(Z_{L y 12}-Z_{L y 22}\right) a_{L 1}=M_{Y} \\
& I_{L 2 x} \ddot{\theta}_{L 2 x}+C_{e q L x 11}\left(\dot{Z}_{L x 21}-\dot{Z}_{L x 11}\right) a_{L 2}-C_{e q L x 12}\left(\dot{Z}_{L x 22}-\dot{Z}_{L x 12}\right) a_{L 2}+C_{e q L x \times 11}\left(\dot{Z}_{L x 21}-\dot{Z}_{L x 31}\right) a_{L 2}-C_{e q L x 22}\left(\dot{Z}_{L x 22}-\dot{Z}_{L x 32}\right) a_{L 2} \\
& +k_{e q L x 11}\left(Z_{L x 21}-Z_{L x 11}\right) a_{L 2}-k_{e q L \times 12}\left(Z_{L x 22}-Z_{L x 12}\right) a_{L 2}+k_{e q L x 21}\left(Z_{L x 21}-Z_{L x>11}\right) a_{L 2}-k_{e q L x 22}\left(Z_{L x 22}-Z_{L x 32}\right) a_{L 2}=0 \\
& I_{L 2 y} \ddot{\theta}_{L 2 y}+C_{e q L y 11}\left(\dot{Z}_{L y 21}-\dot{Z}_{L y 11}\right) a_{L 2}-C_{e q L y 12}\left(\dot{Z}_{L y 22}-\dot{Z}_{L y 12}\right) a_{L 2}+C_{e q L y 21}\left(\dot{Z}_{L y 21}-\dot{Z}_{L y 31}\right) a_{L 2}-C_{e q L y 22}\left(\dot{Z}_{L y 22}-\dot{Z}_{L y 32}\right) a_{L 2} \\
& +k_{e q L y 11}\left(Z_{L y 21}-Z_{L y 11}\right) a_{L 2}-k_{e q L y 12}\left(Z_{L y 22}-Z_{L y 12}\right) a_{L 2}+k_{e q L y 21}\left(Z_{L y 21}-Z_{L y 31}\right) a_{L 2}-k_{e q L y 22}\left(Z_{L y 22}-Z_{L y 32}\right) a_{L 2}=0 \\
& I_{L 3 x} \ddot{\theta}_{L 33 x}+C_{e q L x 21}\left(\dot{Z}_{L x 31}-\dot{Z}_{L x 21}\right) a_{L 3}-C_{e q L L 222}\left(\dot{Z}_{L x 32}-\dot{Z}_{L x 22}\right) a_{L 3}+C_{e q L L 31}\left(\dot{Z}_{L x 31}-\dot{Z}_{r x 1}\right) a_{L 3}-C_{e q L x 32}\left(\dot{Z}_{L x 32}-\dot{Z}_{r x 2}\right) a_{L 3} \\
& +k_{e q L x 21}\left(Z_{L x 31}-Z_{L x 21}\right) a_{L 3}-k_{e q L x 22}\left(Z_{L x 32}-Z_{L x 22}\right) a_{L 3}+k_{e q L x 31}\left(Z_{L x 31}-Z_{r x 1}\right) a_{L 3}-k_{e q L \times 32}\left(Z_{L x 32}-Z_{r x 2}\right) a_{L 3}=0 \\
& I_{L 3 y} \ddot{\theta}_{L 3 y}+C_{e q L y 21}\left(\dot{Z}_{L y 31}-\dot{Z}_{L y 21}\right) a_{L 3}-C_{e q L L 22}\left(\dot{Z}_{L y 32}-\dot{Z}_{L y 22}\right) a_{L 3}+C_{e q L y 31}\left(\dot{Z}_{L y 31}-\dot{Z}_{r y 1}\right) a_{L 3}-C_{e q L y 32}\left(\dot{Z}_{L y 32}-\dot{Z}_{r y 2}\right) a_{L 3} \\
& +k_{e q L y 21}\left(Z_{L y 31}-Z_{L y 21}\right) a_{L 3}-k_{e q L L 22}\left(Z_{L y 32}-Z_{L y 22}\right) a_{L 3}+k_{e q L y 31}\left(Z_{L y 31}-Z_{r y 1}\right) a_{L 3}-k_{e q L y 32}\left(Z_{L y 32}-Z_{r y 2}\right) a_{L 3}=0 \\
& I_{r x} \ddot{\theta}_{x}+C_{e q L x>31}\left(\dot{Z}_{r x 1}-\dot{Z}_{L x 31}\right) a_{r}-C_{e q L x 32}\left(\dot{Z}_{r x 2}-\dot{Z}_{L x 32}\right) a_{r}+C_{e q q x 1}\left(\dot{Z}_{r x 1}-\dot{Z}_{d}\right) a_{r}-C_{e q x \times 2}\left(\dot{Z}_{r x 2}-\dot{Z}_{d}\right) a_{r} \\
& +k_{e q L 331}\left(Z_{r x 1}-Z_{L x 31}\right) a_{r}-k_{e q L 332}\left(Z_{r x 2}-Z_{L x 32}\right) a_{r}+k_{e q q x 1}\left(Z_{r x 1}-Z_{d}\right) a_{r}-k_{e q \times 22}\left(Z_{r x 22}-Z_{d}\right) a_{r}=0 \\
& I_{r y} \ddot{\theta}_{y}+C_{e q L y 31}\left(\dot{Z}_{r y 1}-\dot{Z}_{L y 31}\right) a_{r}-C_{e q L y 32}\left(\dot{Z}_{r y 2}-\dot{Z}_{L y 32}\right) a_{r}+C_{e q y y 1}\left(\dot{Z}_{r y 1}-\dot{Z}_{d}\right) a_{r}-C_{e q y 2}\left(\dot{Z}_{r y 2}-\dot{Z}_{d}\right) a_{r} \\
& +k_{e q L y 31}\left(Z_{r y 1}-Z_{L y 31}\right) a_{r}-k_{e q L y 32}\left(Z_{r y 2}-Z_{L y 32}\right) a_{r}+k_{e q y 31}\left(Z_{r y 1}-Z_{d}\right) a_{r}-k_{e q y 2}\left(Z_{r y 2}-Z_{d}\right) a_{r}=0
\end{aligned}
$$

In dynamic model, the meaning of each parameter is shown below.

$I_{\kappa}(\kappa=L 1, L 2, L 3, r, p i, m i)$-The rotational inertia of each component.

$I_{\kappa x}, I_{\kappa y}(\kappa=L 1, L 2, L 3, r)$ - The radial rotational inertia of each component.

$m_{\kappa}(\kappa=L 1, L 2, L 3, r, p i, d)$-The mass of each component.

$k_{\text {eqk }}(k=x i, y i, L i)$-The radial stiffness of eachcutterheadcomponent.

$k_{\text {eq }}(\kappa=r, r z, d, d z)$-The radial, axial stiffness of bull gear and shield. 
$k_{L 1 L 2}, k_{L 2 L 3}, k_{L 3 r}$-The torsional stiffness of front panel, support rod and flange of cutterhead.

$k_{m p Q}, k_{e q p}, k_{(t)}$-The torsional stiffness of transmission shaft, the support stiffness of pinions, the time-varying meshing stiffness.

$T_{L}, T_{p i}, F_{x}, F_{y}, F_{L}, M_{x}, M_{y}$-The load torque on cutterhead, the input motor torque, horizontal unbalance force, vertical unbalance force, axial force, horizontal overturning moment, vertical overturning moment.

$r_{b r}, \quad r_{b p}$-The base radius of bull gear and pinions.

$\theta_{i}, x_{i}, y_{i}, z_{i}$-The angle, horizontal displacement, vertical displacement and axial displacement of each component.

The nonlinear equations can be established according to the dynamic model.

The solution principle and solving process of Newmark method

The analytical approaches to solving system dynamic equation mainly include perturbation method, harmonic balance method, fourier series method, etc ${ }^{[1]}$. It is difficult to obtain the accurate analytical solution in solving a multi-factor coupling nonlinear dynamic equation. The Newmark method and Rungekutta method are the mostly used methodin engineering.

Newmark method belongs to step by step integral method.The time step length does not affect the solving stability, and the values of this step length can be determined according to the solving precision. Newmark method avoids the error stack effect in solving process, and can adapt to the nonlinear response analysis. Its disadvantage exists in forming the complicated stiffness matrix. The core part of Newmark method is establishing a recursive relationship from $t$ to $t+\Delta t$. Thedisplacement $x_{t+\Delta t}$, velocity $\dot{x}_{t+\Delta t}$ and acceleration $\ddot{\ddot{x}}_{t+\Delta t}$ are unknowns in step $t+\Delta t$.

Newmark method assumes the velocity and displacement are:

$$
\left\{\begin{array}{l}
\dot{x}_{t+\Delta t}=\dot{x}_{t}+\left[(1-\gamma) \ddot{x}_{t}+\gamma \ddot{x}_{t+\Delta t}\right] \Delta t \quad 0 \leq \gamma \leq 1 \\
x_{t+\Delta t}=x_{t}+\dot{x}_{t} \Delta t+\left[\left(\frac{1}{2}-\beta\right) \ddot{x}_{t}+\beta \ddot{x}_{t+\Delta t}\right] \Delta t^{2} \quad 0 \leq 2 \beta \leq 1
\end{array}\right.
$$

The above formulas are the basic formula of Newmark method. Parameters $\gamma$ and $\beta$ are of great influence on the calculation results. The values of $\gamma$ and $\beta$ should be determined fully considering the integral precision and stability. According to the Newmark method, the acceleration of the method is constant when $\gamma \geq 0.5$, $\beta \geq(0.5+\gamma)^{2} / 4$. The value of acceleration is $\frac{1}{2}\left(\ddot{x}_{t}+\ddot{x}_{t+\Delta t}\right)$. method is unconditionally stable.

The differential equation of next step is

$$
[M] \ddot{x}_{t+\Delta t}+[C] \dot{x}_{t+\Delta t}+[K] x_{t+\Delta t}=[F]_{t+\Delta t}
$$

Plug that intointo equation (1), and the equation in step $t+\Delta t$ is obtained.

$$
[\bar{K}] x_{t+\Delta t}=[\bar{F}]_{t+\Delta t}
$$

The $[\bar{K}]$ iseffective stiffness matrix which can be calculated by following equations. 


$$
\begin{gathered}
{[\bar{K}]=[K]+\frac{1}{\gamma \Delta t^{2}}[M]+\frac{\beta}{\gamma \Delta t}[C]} \\
{[\bar{F}]=[F]_{t+\Delta t}+[M]\left(\frac{1}{\gamma \Delta t^{2}}\{u\}_{t}+\frac{1}{\gamma \Delta t}\{\dot{u}\}_{t}+\left(\frac{1}{2 \gamma}-1\right)\{\ddot{u}\}_{t}\right)} \\
+[C]\left(\frac{\beta}{\gamma \Delta t}\{u\}_{t}+\left(\frac{\beta}{\gamma}-1\right)\{\dot{u}\}_{t}+\left(\frac{\beta}{2 \gamma}-1\right) \Delta t\{\ddot{u}\}_{t}\right)
\end{gathered}
$$

According to (3), the displacement of next moment ${ }^{x_{t+\Delta t}}$ can be obtained. The velocity and acceleration of next moment ${ }^{\dot{x}_{t+\Delta t}}, \ddot{\dot{x}}_{t+\Delta t}$ can be calculated by equation (1). Based on the analysis above, the calculation process of Newmarkmethod is shown in figure 2.

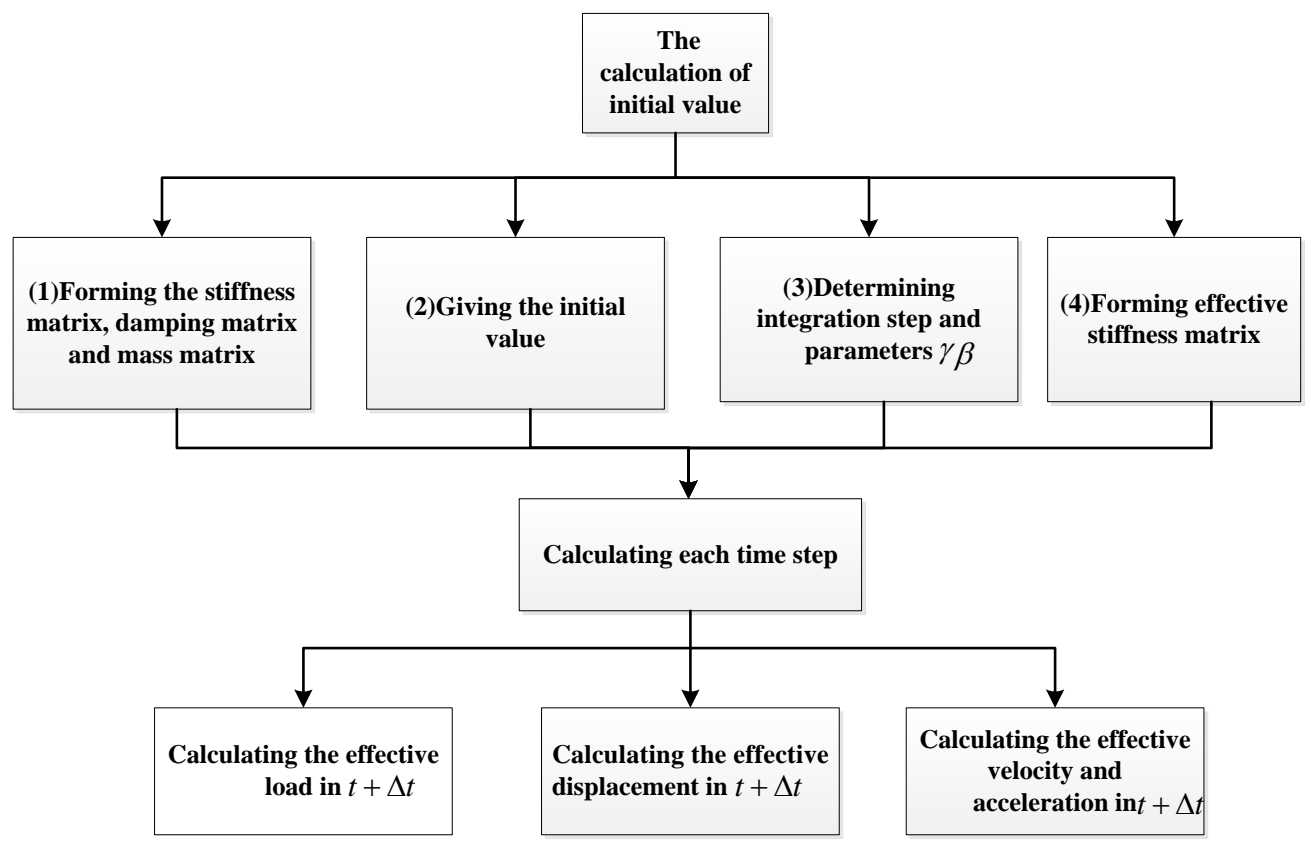

Figure 2. Calculation Process

The calculation process of a complicated dynamic model by Newmark method 3.1 The mass matrix $M$, stiffness matrix $K$, damping matrix $C$ and load matrix $F$. The total mass matrix is a $59 \times 59$ diagonal metrix which is listed below.

$$
\begin{aligned}
M=\operatorname{diag} & \left(I_{m 1} I_{p 1} I_{m 2} I_{p 2} I_{m 3} I_{p 3} I_{m 4} I_{p 4} I_{m 5} I_{p 5} I_{m 6} I_{p 6} I_{m 7} I_{p 7} I_{m 8} I_{p 8}\right. \\
& I_{r} I_{L 3} I_{L 2} I_{L 1} m_{L 1} m_{L 1} m_{L 2} m_{L 2} m_{L 3} m_{L 3} m_{r} m_{r} 2 n_{d} m_{d} m_{p 1} m_{p 2} \\
& m_{p 3} m_{p 4} m_{p 5} m_{p 6} m_{p 7} m_{p 8} m_{p 1} m_{p 2} m_{p 3} m_{p 4} m_{p 5} m_{p 6} m_{p 7} m_{p 8} \\
& \left.m_{L 1} m_{L 2} m_{L 3} m_{r} m_{d} I_{L 1 x} I_{L 1 y} I_{L 2 x} I_{L 2 y} I_{L 3 x} I_{L 3 y} I_{r x} I_{r y}\right)
\end{aligned}
$$

Stiffness matrix $K$ is the most complicated factor that influences the system's dynamic characteristics. The stiffness matrix may contain time-varying stiffness element which make the whole matrix nonlinear.

In the numerical example, the stiffness elements of torsional and radial DOF are timevarying. There are $20 \mathrm{DOF}$ in torsional direction while radial direction contains $26 \mathrm{DOF}$. Therefore, the first 46 orders of the stiffness matrix is a multi-DOF coupling time-varying 
matrix. This time-varying matrix is defined as $k 111$, and its corresponding damping matrix is defined as $c 111$. The last 13 orders of the stiffness matrix represent the stiffness of each component in axial and capsized DOF, and the corresponding stiffness matrix and damping matrix are named $k 222$ and $c 222$.The system stiffness matrix is defined as:

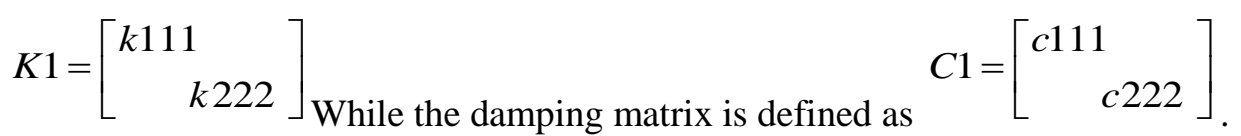

The load matrix contains the external load, time-varying meshing load, cumulative error, etc. It is a complicated vector with time-varying characteristic, and is listed below.

$$
\begin{aligned}
& F=\left[T_{p i},-r_{b p} *\left(f_{N i}+D_{N i}\right), r_{b r} \cdot \sum_{i=1}^{8}\left(f_{N i}+D_{N i}\right), 0,0,-T_{L}, F X, F Y, 0,0,0,0,\right. \\
& \sum_{i=1}^{8}\left(-f_{N i}+D_{N i}\right) \cdot \sin \left(\varphi_{i}+\alpha\right), \sum_{i=1}^{8}\left(f_{N i}+D_{N i}\right) \cdot \cos \left(\varphi_{i}+\alpha\right),-\left(f_{N i}+D_{N i}\right) \cdot \cos \alpha, \\
& \left.-\left(f_{N i}+D_{N i}\right) \cdot \sin \alpha, F L, 0,0,0,0, M X, M Y, 0,0,0,0,0,0\right]^{T}
\end{aligned}
$$

1.2 Integration step and parameters $\gamma$ and $\beta$.

In order to make the Newmark method unconditionally stable, the integration parameters are selected and calculated as follows.

$$
\begin{gathered}
\Delta t=0.02, \gamma=0.5, \beta=1 / 4, \alpha_{0}=\frac{1}{\beta \Delta t^{2}}, \alpha_{1}=\frac{\gamma}{\beta \Delta t} \\
\alpha_{2}=\frac{1}{\beta \Delta t}, \alpha_{3}=\frac{1}{2 \beta}-1, \alpha_{4}=\frac{\gamma}{\beta}-1, \alpha_{5}=\frac{\Delta t}{2}\left(\frac{\gamma}{\beta}-2\right), \alpha_{6}=\Delta t(1-\gamma), \alpha_{7}=\gamma \Delta t
\end{gathered}
$$

1.3 The calculation of effective stiffness matrix

The effective stiffness matrix is calculated as equation below.

$$
\bar{K}=K+\alpha_{0} M+\alpha_{1} C
$$

1.4 Calculation for each time step

The system effective load vector is computed as:

$$
\bar{F}_{t+\Delta t}=F_{t+\Delta t}+M\left(\alpha_{0} x_{t}+\alpha_{2} \dot{x}_{t}+\alpha_{3} \ddot{x}_{t}\right)+C\left(\alpha_{1} x_{t}+\alpha_{4} \dot{x}_{t}+\alpha_{5} \ddot{x}_{t}\right)
$$

The displacement in moment $t+\Delta t$ is calculated as:

$$
\bar{K} x_{t+\Delta t}=\bar{F}_{t+\Delta t}
$$

The velocity and acceleration in moment $t+\Delta t$ is calculated as:

$$
\left\{\begin{array}{l}
\ddot{x}_{t+\Delta t}=\alpha_{0}\left(x_{t+\Delta t}-x_{t}\right)-\alpha_{2} \dot{x}_{t}-\alpha_{3} \ddot{x}_{t} \\
\dot{x}_{t+\Delta t}=\dot{x}_{t}+\alpha_{6} \ddot{x}_{t}+\alpha_{7} \ddot{x}_{t+\Delta t}
\end{array}\right.
$$

The comparative analysis of Newmark and Rungekutta method:

For this dynamic system, the displacement, velocity and acceleration calculation results by Newmark and Rungekutta method are show in figure 3.In figure, curve $\mathrm{S}$ represents the displacement response, curve $\mathrm{V}$ represents the velocity response and curve a represents the acceleration response. 


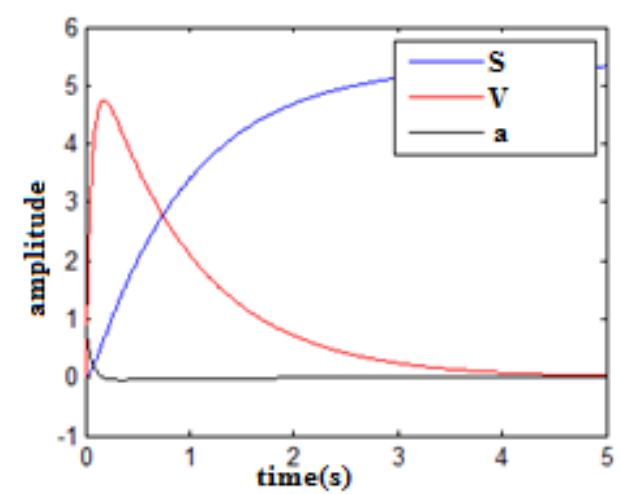

(A) Runge-Kutta Method

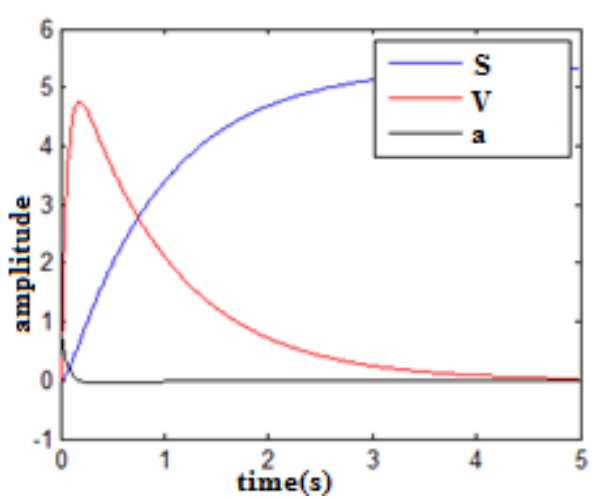

(B) Newmark Method

Figure 3. Solving Results

From the figure 2, it can be seen that the response results by these two methods are in strong consistency which ensures the solving results are in the same precision.

The response displacementbyNewmark method in different integration steps are shown in Figure 4.

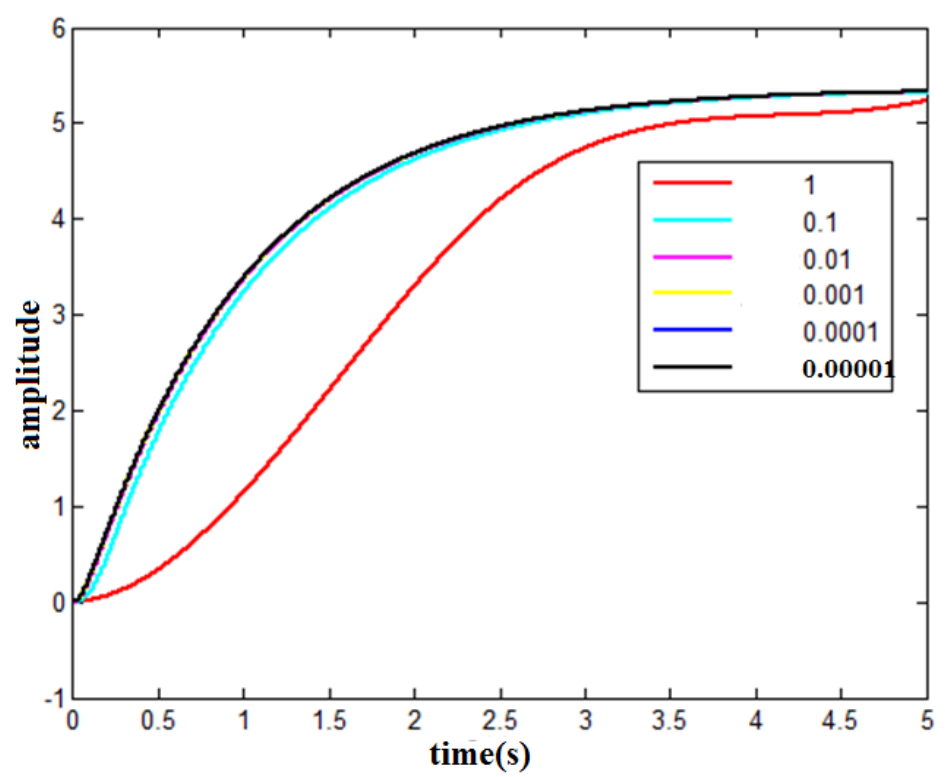

Figure 4. Solving Step to the Results

It can be seen that the response results become stable with the integration steps reducing from 1 to 0.00001 . Especially when integration step reduces from 1 to 0.1 , the response results tend towards the analytical solution rapidly. 1.

The computing time and memory statistics using these two methods are listed in Table

Table 1. Contrast of the Calculation Statistics

\begin{tabular}{cccc}
\hline Method & $\begin{array}{c}\text { Truncation } \\
\text { error }\end{array}$ & $\begin{array}{l}\text { Solving } \\
\text { time }\end{array}$ & $\begin{array}{c}\text { Memory } \\
\text { statistics }\end{array}$ \\
\hline Rungekutta & $O\left(\Delta t^{3}\right)$ & $2.6 \mathrm{e} 5$ & 503 \\
Newmark & $O\left(\Delta t^{3}\right)$ & 155 & 1167 \\
Ratio & 1 & 0.0006 & 2.32 \\
\hline
\end{tabular}


As can be seen above, the Newmark method shortens the solving time by at least 3 orders comparing that of Rungekutta method. Therefore, applying Newmark method to the nonlinear time-varying system can largely shorten the solving time.

\section{Conclusion}

The Newmark and Rungekuttamethod are used in calculating a same complex nonlinear dynamic equation. The results show that when integration step reduces from 1 to 0.1 , the response results tend towards the analytical solution rapidly, and the response results become stable with integration step continues reducing. Moreover, the Newmark method shortens the solving time by at least 3 orders comparing that of Rungekutta method. These results provide the theory basis for the selection of numerical integration method solving complex dynamic equation.

\section{Acknowledgments}

This project is supported by National Natural Science Foundation of China (Grant No. 51375001, 51309045).

\section{References}

[1] J. C. Butcher Univ, "The numerical analysis of ordinary differential equations", New York: WileyInterscience, (1987)

[2] Q. Fang and Z. Chen, “Accuracy of explicit Newmark Scheme for wave propagation problems", Chinese Journal of Geotechnical Engineering, vol. 15, no. 1, (1993).

[3] H. Li and T. Wang, "An interpretation on Newmark beta methods in mechanism of numerical analysis", Journal of Earthquake Engineering and Engineering Vibration, vol. 31, no. 2, (2011), pp. 55-62.

[4] W. Zai, "The explicit scheme of Newmark's integration method for large structural dynamic analysis", Journal of Chongqing Jiaotong Institute, vol. 10, no. 2, (1991), pp. 33-40.

[5] S. He and X. Wu, "A shock simulation method for rotor systems-average velocity newmark-riccati transfer matrix technique", Chinese Journal of Applied Mechanics, vol. 27, no. 4, (2010), pp. 823-829.

[6] S. Li, Y. Cai and C. Xu, "Time step length determination of Newmark method for Dynamic responses of railway tracks", Journal of Zeijiang University, vol. 48, no. 2, (2014), pp. 228-234.

[7] Z. Gu and S. Che, "Prohl transfer matrix Newmark formulation integration method for rotor dynamics", Journal of Vibration Engineering, vol. 3, no. 3, (1990), pp. 82-91.

[8] X. Yufeng and G. Jing, "A Self-adaptive Newmark method with parameters dependent upon structure dynamic characteristics", Chinese Journal of Theoretical and Applied Mechanics, vol. 44, no. 5, (2012), pp. 904-911.

[9] W. Sun, J. Ling and J. Huo, "Dynamic characteristics study with multidegree-of-freedom coupling in TBM cutterhead system based on complex factors", Mathematical Problems in Engineering, (2013).

[10] C. Jin, "Dynamic Performance Analysis and Parameter Optimization of Wind Power Speed-up Machine Planetary Gear Trains", Dalian: Dalian University of Technology, (2012).

[11] R. Li and J. Wang, "Gear transmission system dynamics”, Beijing: Science Press, (1997), p. 3.

[12] Promotion Center for Science \& Technology Achievements of Ministry of Water Resources, Full face rock tunnel boring machine (TBM), Beijing: Petroleum Industry Press, (2005).

\section{Authors}

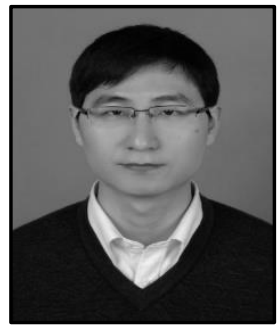

Junzhou Huo is currently a master supervisor at the School of Mechanical Engineering at Dalian University of Technology in China. Huo's research interests include layout optimization and TBM cutter head design. 

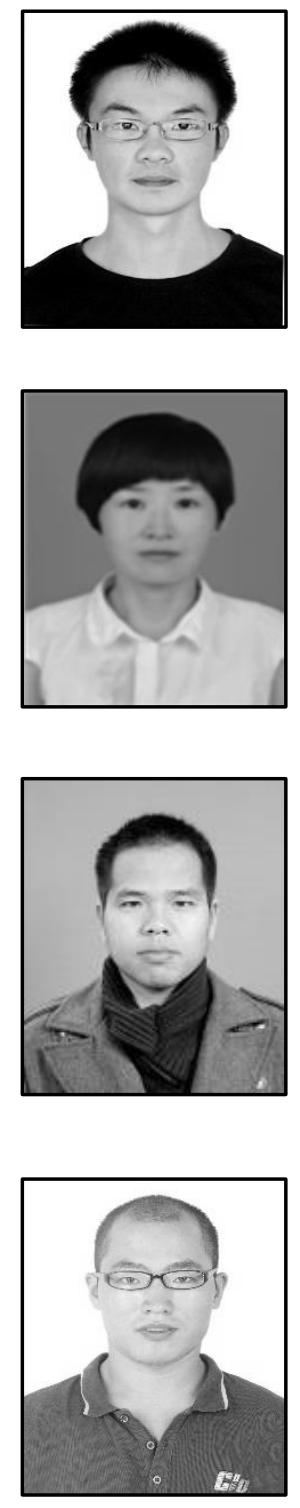

Hanyang Wu is currently a master at the School of Naval Architecture Engineering at Dalian University of Technology in China. Wu's research interests include optimization design.

Jing Chen is currently an assistant professor in the School of Naval Architecture and Ocean Engineering in Dalian Ocean University. Her current research interests include ships' subdivision design, loading design, stability and strength design, and their applications in ship design and shipping.

Jing-Xiu Ling is currently a Ph.D. candidate at the School of Mechanical Engineering at Dalian University of Technology in China. Ling's research interests include system dynamics and life prediction of TBM cutter head.

Guang-Qing $\mathbf{L i}$ is currently a master at the School of Mechanical Engineering at Dalian University of Technology in China. Li's research interests include TBM cutter head testbench design and TBM cutter head design. 
International Journal of Hybrid Information Technology Vol.8, No.7 (2015) 